\title{
Application of Boyd's Periodization and Relaxation Method in a Spectral Atmospheric Limited-Area Model. Part I: Implementation and Reproducibility Tests
}

\author{
PiET TERMONIA \\ Royal Meteorological Institute, Brussels, and Department of Physics and Astronomy, Ghent University, Ghent, Belgium \\ FABRICE VOITUS \\ CNRM, Météo-France, Toulouse, France \\ DAAN DEGRAUWE \\ Royal Meteorological Institute, Brussels, Belgium \\ STEVEN CALUWAERTS \\ Department of Physics and Astronomy, Ghent University, Ghent, Belgium \\ RAFIO HAMDI \\ Royal Meteorological Institute, Brussels, Belgium
}

(Manuscript received 31 January 2012, in final form 6 April 2012)

\begin{abstract}
This paper describes the implementation of a proposal of Boyd for the periodization and relaxation of the fields in a full three-dimensional spectral semi-implicit semi-Lagrangian limited-area model structure of an atmospheric modeling system called HARMONIE that is used for numerical weather prediction and regional climate studies. Some first feasibility tests in an operational numerical weather prediction context are presented. They show that, in terms of standard operational forecast scores, Boyd's windowing-based method provides comparable performance as the old existing spline-based periodization procedure. However, the real improvements of this method should be expected in specific cases of strong dynamical forcings at the lateral boundaries. An extensive demonstration of the superiority of this windowing-based method is provided in an accompanying paper.
\end{abstract}

\section{Introduction}

Fourier spectral limited-area models (SLAMs) are presently common practice for operational numerical weather prediction (NWP) applications. Many national weather forecast centers have decided to exploit the accuracy and computational efficiency the Fourier spectral technique offers for high-order derivatives, in comparison to traditional finite-differences gridpoint methods. For example, the Japan Meteorological Agency (JMA; Tatsumi 1986), the National Centers for Environment Prediction

Corresponding author address: Piet Termonia, Royal Meteorological Institute, Ringlaan 3, B-1180 Brussels, Belgium.

E-mail: piet.termonia@meteo.be
(NCEP; Juang 1992), the High-Resolution Limited-Area Model (HIRLAM; Haugen and Machenhauer 1993), and the Aire Limitée Adaptation Dynamique Développement International (ALADIN; ALADIN International Team 1997) are SLAMs that are used operationally. The ALADIN model is currently also used for regional climate studies (e.g., see Radu et al. 2008; Hamdi et al. 2012).

When imposing the lateral boundaries in a limitedarea model essentially two problems have to be addressed. The first is the mathematical formulation of the lateral boundary conditions (LBCs). A second difficulty is that time-dependent LBCs must be imposed during the forecast. In practice one relies on temporally interpolated data, usually coming from a global model and this sometimes creates artificial dual low structures (Tudor and 
Termonia 2010; Termonia et al. 2011) near the lateral boundaries, potentially leading to large errors in rare but crucial storm cases (see Termonia 2003; Termonia et al. 2009). Some methods exist to address this. In particular, such temporal resolution problems can be detected and quantified (see Termonia 2004), and some techniques have been proposed to deal with the resulting errors (Termonia et al. 2011).

Running a Fourier spectral model implies solving an extra difficulty. The use of the Fourier series to approximate a nonperiodic flow requires that the fields are made periodic on the limited-area domain. The present paper deals with this problem.

All NWP Fourier SLAMs have been derived following the same basic idea: periodization and blending with low-resolution global data. Indeed, the dual constraints of the periodicity of the fields defined on the LAM domain and the LBCs are currently addressed through heuristic periodization procedures combined usually with a Davies relaxation scheme (Davies 1976, 1983) for blending high-resolution limited-area data with lowerresolution global data. Different methods to construct periodic fields out of the nonperiodic ones have been developed. The Fourier extension method proposal of Haugen and Machenhauer has been adopted with some minor technical differences by the HIRLAM and ALADIN models; Tatsumi's sinusoidal-subtracted sine cosine variable separation method was used for the JMA model; and the perturbation method, which is a variant of Tatsumi's method, was deployed in the NCEP model, as advocated by Hoyer (1987).

However, the various periodization procedures used by all above-mentioned operational SLAMs are based on empiricism and pragmatism rather than on solid mathematical grounds, and may thereby suffer from serious deficiencies, as noted by Laprise (2003). One major criticism is that the high-order derivatives remain discontinuous, which causes a slow convergence of the double Fourier expansion series and jeopardizes the high-order accuracy of the entire Fourier spectral method, as discussed in Boyd (2002) and Laprise (2003). In addition, it has been argued by Kuo and Williams (1992) that the Tatsumi-type method may cause spurious oscillations at lateral boundaries when large disturbances travel across the limited-area borders. Besides, regarding the HaugenMachenhauer-type methods, Gustafson et al. (2001) pointed out that the formulation of Fourier extension zone may have a noticeable impact on the data assimilation scheme through the calibration of the background error-covariance matrix B. Therefore, it seems relevant to explore some new idea for periodization and relaxation that might overcome the main drawback of both the Tatsumi and Haugen-Machenhauer method.
Boyd (2005) proposed a new periodization and relaxation procedure that ensures both periodicity and blending while preserving high-order Fourier spectral accuracy. Essentially based on a Fourier extension method of the limited-area domain, Boyd's innovation consists of the use of infinitely differentiable "windowing functions" generally employed in wavelet theory, both to make the LAM fields periodic on the extended domain and to relax these fields toward the large-scale global solution at the lateral boundaries. In what follows, we will refer to this as the windowing-based method. Promising results were presented in that paper, but only for the academic one-dimensional Burgers' equation discretized according to an Eulerian explicit scheme [i.e., for a small CourantFriedrichs-Lewy (CFL) number]. Actually, Boyd asserts that with his method that "The extension and Davies relaxation produces no errors unless the time step is an order of magnitude larger than the CFL limit" (Boyd 2005, p. 2038). However, operational NWP models often use a semi-implicit semi-Lagrangian formulation that allows for substantially larger time steps. Moreover, it remains an open question whether Boyd's proposal remains an attractive approach in the presence of dynamical forcing terms like orography or physical processes. For these reasons, further experiments have to be carried out before considering windowing-based periodization and relaxation as a reliable alternative for NWP purposes.

This paper describes the implementation of the windowing-based method in a state-of-the-art limited-area model and demonstrates that the existing performance of the model can be reproduced in terms of verification scores in an operational NWP environment. Because some aspects of the periodization procedure of the ALADIN SLAM are quite similar to the one proposed by Boyd (viz., the need for an additional Fourier extension zone), we have made the choice to test the windowing-based method in the framework of the ALADIN spectral model. Section 2 therefore provides a summarizing description of the ALADIN model, with a focus on the aspects of the model that are altered by this new alternative. Section 3 describes the windowing-based method itself and emphasizes the implications of operational NWP requirements on the windowing-based periodization and relaxation procedures. The results of operational-like experiments are presented in section 4 and we finish this paper with some concluding remarks in section 5 .

Substantial improvements due to the new method in the SLAM, if they exist, should be expected to happen in situations of very strong dynamical forcings at the lateral boundaries, for instance, in relatively rare cases of strong incoming storms. But a detailed analysis of the effects of the application of the windowing-based periodization procedure requires a careful setup of the experiments, which 
needs to take into account other issues as well, in particular the temporal-resolution problem. This falls outside the scope of this paper, but it is presented in an accompanying paper (Degrauwe et al. 2012, hereafter Part II).

\section{Model description}

This section describes the implementation of Boyd's windowing-based method within the hydrostatic version of the dynamical kernel of the ALADIN model (ALADIN International Team 1997).

ALADIN is a spectral limited-area mesoscale NWP model built in cooperation between the national meteorological services of 16 European and Northern African countries, through a consortium structure. This model is also the limited-area version of the global Integrated Forecast System (IFS) of the European Centre for Medium-Range Weather Forecasts (ECMWF) and the Action de Recherche Petite Echelle Grande Echelle (ARPEGE) model of Météo-France and used operationally in these two meteorological centers. The nonhydrostatic $(\mathrm{NH})$ and hydrostatic primitive equations (HPE) versions of the ALADIN dynamical kernel are implemented in a single software package.

Over the past decade, the dynamical kernel of this model has been used to develop two new model configurations, the so-called ALARO model and the Applications of Research to Operations at Mesoscale (AROME) model. Both share the above mentioned kernel with its HPE and $\mathrm{NH}$ options, but call different options of the physics parameterizations. The ALARO physics parameterization is developed with the aim to make the model suitable to run at resolutions in the so-called gray zone (i.e., in the range from 3 to $7 \mathrm{~km}$ ). It includes the $3 \mathrm{MT}$ parameterization of deep convection based on research by Gerard and Geleyn (2005), Gerard (2007), and Gerard et al. (2009), the Ritter-Geleyn scheme for radiation (Ritter and Geleyn 1992), a semi-Lagrangian horizontal diffusion scheme called SLHD (Váña et al. 2008), some pseudoprognostic turbulent kinetic energy (TKE) scheme (pTKE), and a statistical sedimentation scheme for precipitation (Geleyn et al. 2008). The coupling to the dynamical kernel is based on a flux-conservative formulation of the equations proposed by Catry et al. (2007). An extensive description of the AROME model is given by Seity et al. (2011). Thus, three different model configurations are implemented in one single model code. This code is currently also used and further developed by the European HIRLAM consortium, and the system incorporating all those configurations is referred to as the HIRLAM-ALADIN Research on Mesoscale Operational NWP in EuroMediterranean Partnership (Euromed) (HARMONIE) system. The implementations described in the present paper are available to all model configurations in this system, and we will use the terminology HARMONIE system or HARMONIE, whenever it is necessary to stress this. We will, however, still refer to the common dynamical kernel as the ALADIN dynamical kernel when referring to that part of the kernel that was developed in the past within the ALADIN consortium.

Currently, several versions of ALADIN and ALARO are exploited daily in the various national meteorological services of the ALADIN consortium at resolutions of about $5-10 \mathrm{~km}$, using the HPE version of the model with the following characteristics: a horizontal spectral representation of the fields based on a double Fourier expansion assuming a Fourier extension zone to periodize the fields, a mass-based hybrid terrain-following vertical coordinate $\eta$ (Simmons and Burridge 1981; Laprise 1992), and a two-time-level semi-implicit semiLagrangian (2-Tl SISL) time discretization (Temperton et al. 2001), using the so-called Stable Extrapolation Two-Time-Level Scheme (SETTLS; Hortal 2002), to control fast dynamical processes and to deal with advection. The ALADIN model, and by extension all the HARMONIE configurations, is coupled in a one-way noninteractive manner to its global model counterpart ARPEGE, the IFS of the ECMWF, or an intermediate ALADIN version in a double-nesting procedure. In the present paper will we use the terminology of host model for the model that provides the coupling data, and we will call the coupled model the guest model.

Since the primary goal of this paper is to test the use of the windowing-based method in the HARMONIE framework, the model equations and physics will not be detailed in this section, instead see Bubnová et al. (1995) and Bénard et al. (2010) for more details. However, particular attention will be paid to the periodization, 2-TL SISL, and the coupling relaxation techniques presently used in the HARMONIE models, since these will be affected by the windowing-based method.

\section{a. Domain structure and biperiodization by splines}

The existing biperiodization procedure of the HARMONIE system is based on the Fourier extension method proposed by Haugen and Machenhauer. Their solution consists of an extension of the integration area by an artificial meteorologically meaningless zone (hereafter called the $E$ zone). An example of such a domain structure can be found in Fig. 1, where the validation domain of the presented work is shown. So, the horizontal domain structure is organized in three distinct regions $-C$ zone, $I$ zone, and $E$ zone-synthetically illustrated in a one-dimensional manner in Fig. 2. The $C$ zone represents the region of meteorological interest, the $I$ zone is an intermediate region surrounding the $C$ zone, and 


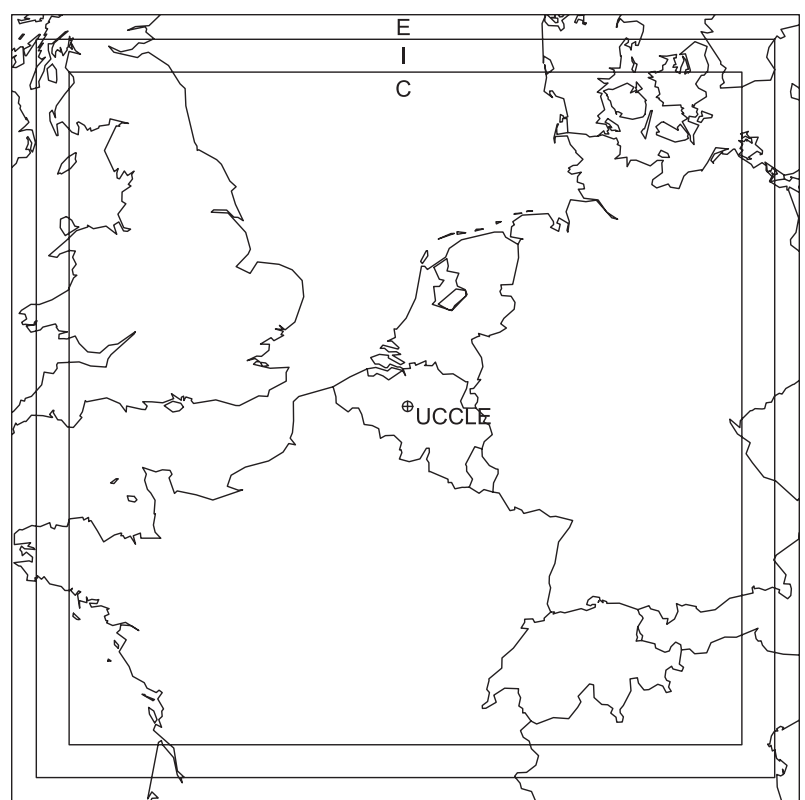

FIG. 1. Domain used for validation with ALARO. The $C$ zone is $164 \times 164$ grid points, the $(C+I)$ zone is $180 \times 180$ grid points, and the $(C+I+E)$ zone is $192 \times 192$ grid points.

dedicated to coupling purposes. We introduce the notation $I_{L}$ and $I_{R}$, indicating the left and the right $I$ zone, as indicated in Fig. 2. In Fig. 1, the $(C+I)$ zone thus corresponds to the integration area, where the nonperiodic meteorologically meaningful fields (i.e., initial data, boundary fields) are known. The purpose of the $E$ zone (which in $1 \mathrm{D}$ again consists of a left and a right part $E_{L}$ and $\left.E_{R}\right)$ is to accommodate extensions of the dynamical fields to make them periodic over the whole $(C$ $+I+E$ ) domain. Typically, the width of the $I_{L}$ and $I_{R}$ zones, in the application of the HARMONIE system, is 8 grid points, while the width of the $E$ zone is 11 grid points. The number of grid points in the $C$ zone is chosen such that the total number of grid points in the $x$ direction and the $y$ direction of the extended $(C+I+E)$ domain are, respectively, numbers factorizable as $2^{k} 3^{l} 5^{m}$ in order to increase the efficiency of the fast Fourier transforms.

For the periodization, as it always existed before implementing the window-based method, only data from the inner $(C+I)$ zone are needed for the construction of the $E$ zone. The calculation of the field values in the $E$ zone is done in two stages. First, extrapolations are done along each row of grid points (corresponding to the $x$ direction) and then along each column of the gridpoint values (thus representing the $y$ direction). Cubic-spline-polynomial functions are used for each one-dimensional extrapolation. The required four coefficients are determined from the demand of

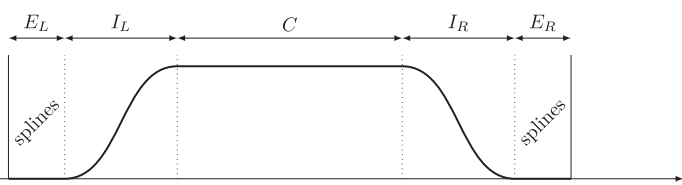

FIG. 2. Organization of the ALADIN domain: central zone $C$, coupling zone $I=I_{L} \cup I_{R}$, and extension zone $E=E_{L} \cup E_{R}$. The solid line represents the relaxation function $\alpha$.

first-order continuity at the beginning and the end of the spline. The second stage of the biperiodization consists of a nine-point transversal smoothing procedure on each grid point lying within the $E$ zone to eliminate noise possibly generated by the one-dimensional row-by-row and column-by-column extrapolations. The mathematical details of this periodization are, for completeness, provided in the appendix. This entire biperiodization procedure will be called the spline-based method hereafter.

The initial data and the 3-hourly lateral boundary fields are rendered biperiodic in advance through this spline-based procedure, before running the model when the coupling fields are created. This is done in the following order: first the fields provided by the host model are interpolated to the grid of the $(C+I)$ zone, then these fields are periodically extended in the $E$ zone with the spline method and finally these fields are transformed with a bi-Fourier transform to spectral space. These spectral coefficients are stored in the coupling files. In other words, the coupling files contain the information of the fields on the entire $(C+I+E)$ zone. During the model runs, at the time of the coupling updates, the coupling data are read from these coupling files, so the corresponding fields are always periodic by construction. On the time steps between the 3-hourly coupling updates they are temporally interpolated between the coupling update times. Please note that this is possible since the interpolation in time between two spatially biperiodic fields yields again a biperiodic field. And thus, during the model run, the model, by construction, always uses biperiodic fields.

Finally, it should also be mentioned that an elliptic spectral truncation is applied to all spectral fields in HARMONIE, in order to remove scales that are beyond the physical model resolution.

\section{b. Time-discretized 2-TL SISL system}

By relaxing most computational stability constraints on advection and fast adjustment processes, two-timelevel semi-implicit semi-Lagrangian (2-TL SISL) schemes allow the use of large time steps. To describe symbolically the ALADIN 2-TL SISL system, the same notations as Bénard et al. (2010) are adopted herein. Here $\mathbf{X}$ is the state vector, $\mathcal{M}$ is the complete dynamical model 
operator, $\mathcal{L}^{*}$ is the linear associated operator, $\mathcal{F}$ is the physical sources operator, and $\Delta t$ is the time step. The 2-TL SISL system to be solved on the limited-area integration domain (without taking into account the coupling process) can be written under the following form:

$$
\begin{aligned}
\left(\mathcal{I}-\frac{\Delta t}{2} \mathcal{L}^{*}\right) \mathbf{X}_{F}^{+}= & \left(\mathbf{X}+\frac{\Delta t}{2} \mathcal{M} \mathbf{X}+\Delta t \mathcal{F}\right)_{O}^{0} \\
& +\frac{\Delta t}{2}\left[\left(\mathcal{M}-\mathcal{L}^{*}\right) \mathbf{X}^{0}-\left(\mathcal{M}-\mathcal{L}^{*}\right) \mathbf{X}^{-}\right]_{o} \\
& +\frac{\Delta t}{2}\left(\mathcal{M}-\mathcal{L}^{*}\right) \mathbf{X}_{F}^{0}
\end{aligned}
$$

where the superscripts $(+, 0,-)$ correspond to values at times $(t+\Delta t, t, t-\Delta t)$, respectively. The subscripts $F$ and $O$ denote the evaluation at the final grid point and the interpolation at the departure point of the SL trajectory, respectively. The SL trajectories are determined using explicitly known winds extrapolated in time.

One feature of the ALADIN semi-Lagrangian scheme relevant for this work is semi-Lagrangian trajectory truncation. This means that SL trajectories are truncated at the lateral boundaries when the origin point is outside the integration domain [i.e., the $(C+I)$ region]. The SL trajectory truncation is an inevitable choice; because of the spline-based extrapolation procedure, the $E$ zone is not supposed to be meteorologically relevant.

Let us denote by $\tilde{\mathbf{X}}_{G}^{+}$the explicit right-hand-side terms of Eq. (1), where the subscript $G$ stands for "guess." It is the result of all dynamical and physical gridpoint (GP) computations, done only over the integration area $(C+$ I). Assuming that $\tilde{\mathbf{X}}_{G}^{+}$is biperiodic over the extended domain $(C+I+E)$, which is actually taken care of by the coupling procedure, see section $2 c$, it is then transformed to spectral space. The completion of the SI time step is done by solving the system in Eq. (1) as

$$
\mathbf{X}^{+}=\left(\mathcal{I}-\frac{\Delta t}{2} \mathcal{L}^{*}\right)^{-1} \tilde{\mathbf{X}}_{G}^{+}
$$

where the subscript $F$ has been dropped for convenience. The advantage of a spectral model like HARMONIE is that the inversion of the operator $\left[\mathcal{I}-(\Delta t / 2) \mathcal{L}^{*}\right]$ is trivial in spectral space. In practice, this is done by reformulating Eq. (2) in the form of a Helmholtz equation whose differential operator becomes diagonal in spectral space. The details of this are not relevant for the present paper and will be not be elaborated on here.

\section{c. Coupling procedure}

The coupling procedure in HARMONIE is achieved by applying a Davies relaxation scheme at the lateral boundaries. In the conventional approach, relaxation is applied at the end of each time step. This is the way in which the boundaries are updated in a purely gridpoint LAM. For a spectral LAM, on the other hand, such a procedure would be quite expensive as it would require an additional inverse and direct spectral transformation. To avoid this computational surplus, the boundary relaxation is performed when the fields are available during the time step in gridpoint space; in the HARMONIE system this happens immediately after all the other GP computations have been completed, as suggested by Radnóti (1995). The coupled version of the preceding 2-TL SISL system in Eq. (1) can then be symbolically written as

$$
\left(\mathcal{I}-\frac{\Delta t}{2} \mathcal{L}^{*}\right) \mathbf{X}^{+}=\alpha \tilde{\mathbf{X}}_{G}^{+}+(1-\alpha)\left(\mathcal{I}-\frac{\Delta t}{2} \mathcal{L}^{*}\right) \mathbf{X}_{H}^{+},
$$

where $\alpha$ denotes the relaxation function varying from zero in the $E$ zone to unity in the $(C+I)$ zone, and $\mathbf{X}_{H}^{+}$ ( $H$ stands for host) represents the large-scale lateral boundary fields at time $t+\Delta t$, typically provided by the global model ARPEGE.

This coupling procedure has two considerable advantages. First, no GP computations are required in the $E$ zone since the contribution of $\tilde{\mathbf{X}}_{G}^{+}$is restricted to the $(C+I)$ area. The $E$ zone contains only the contribution of the large-scale boundary field $\mathbf{X}_{H}^{+}$because the relaxation function $\alpha$ is equal to 0 in the $E$ zone. Second, no application of the biperiodization procedure is required during the course of the integration. The field $\mathbf{X}_{H}^{+}$ is already periodic by construction in the $(C+I+E)$ domain, because it is computed by a simple time interpolation between typically 3-hourly updated largescale states that have been previously made periodic, as outlined before in section $2 \mathrm{a}$. Therefore, the right-handside term of Eq. (3) is already periodic over $(C+I+E)$ and ready to be transformed to spectral space in order to solve the SI system.

The relaxation function $\alpha$ used in the HARMONIE models is given by the following function:

$$
\alpha=1-(p+1) z^{p}+p z^{p+1},
$$

where $z$ varies from 0 to 1 and represents the relative distance inside the $I$ zone. The parameter $p$ has been tuned to a value of 2.16 in order to minimize the reflections at the boundaries. Figure 2 schematically shows the relaxation function on an ALADIN domain.

Although some work has been carried out to find more accurate mathematical techniques for imposing the LBCs in spectral models (Termonia and Voitus 


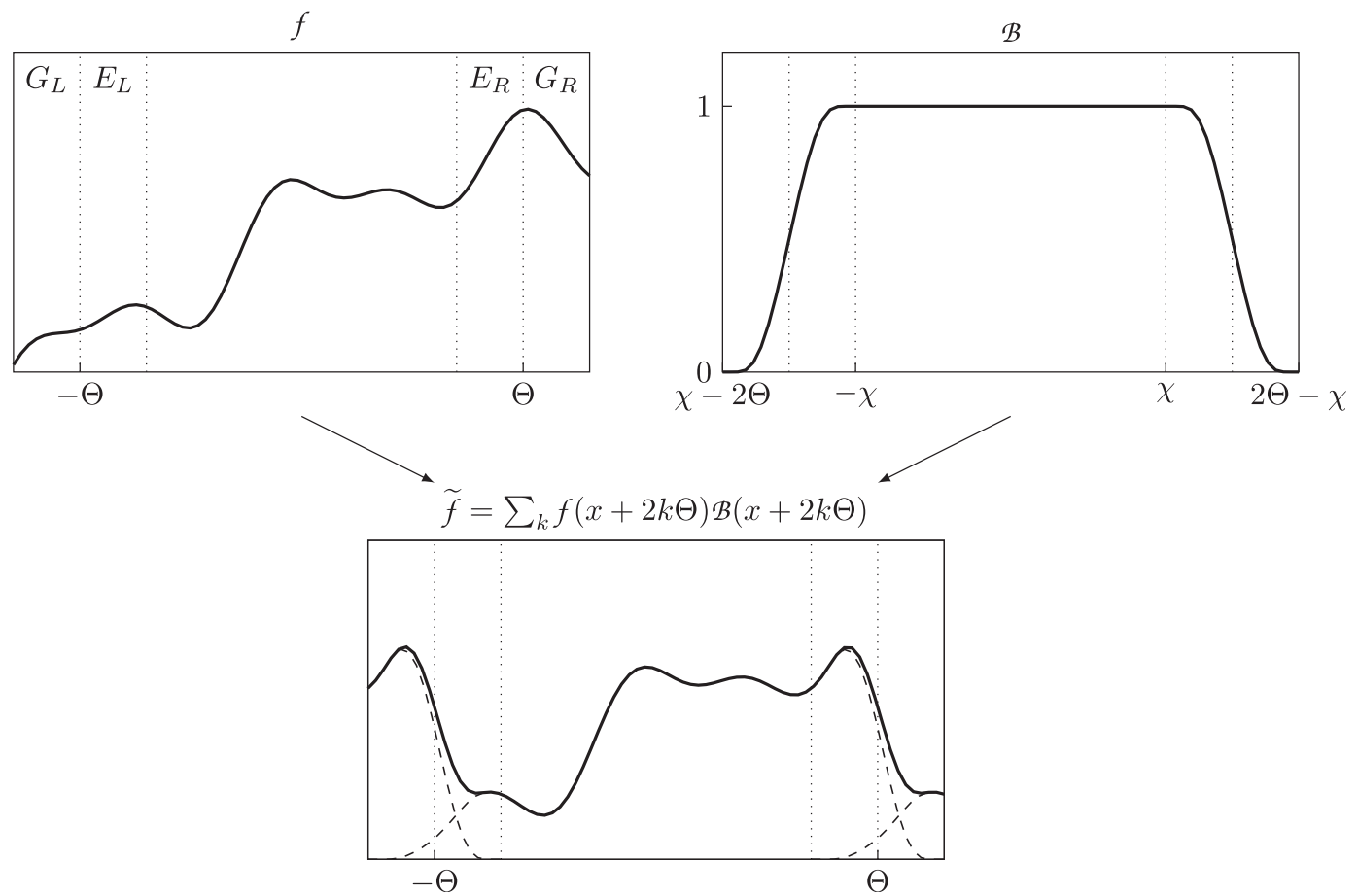

FIG. 3. Boyd's periodization procedure: a periodized function $\tilde{f}$ is obtained on the interval $[-\Theta, \Theta]$ as a summation of shifted multiplications of the nonperiodic function $f$ with a bell window $\mathcal{B}$.

2008; Voitus et al. 2009), so far one has not been able to find a better method than the Davies scheme. Therefore, the work in the present paper will be based on the familiar Davies relaxation and can be seen as an extension thereof.

\section{d. Time-step organization}

Starting from $(C+I+E)$ area-extended and spectrally truncated initial fields in spectral space, the following sequence of calculations is carried out for each time step of the HARMONIE spectral model:

1) Calculation of the horizontal derivatives in spectral space and inverse Fourier transform to GP space.

2) The GP calculations including nonlinear dynamics, determination of trajectories, semi-Lagrangian interpolations and physical parameterizations. These are combined into the right-hand-side terms of the SI equations. This is only done in the $(C+I)$ zone.

3) Boundary relaxation of the right-hand-side terms of the SI equations is done in the way described in section $2 \mathrm{c}$ so that the resulting coupled quantities are biperiodic over the $(C+I+E)$ zone.

4) Fourier transformation of the right-hand-side terms of the SI equations.

5) Solution of the SI equations in spectral space.

6) Implicit horizontal diffusion and time filtering in spectral space.
It can be seen from this procedure that the Davies procedure naturally fits in the time step under step 3 and that this is consistent with using temporally interpolated fields from the periodic coupling fields provided from the host model at the coupling update intervals in the entire $(C+I+E)$ zone.

\section{Boyd's proposal for periodization and relaxation}

In this section, we will first describe the windowingbased method proposed by Boyd. Afterward we will focus on the implications of this method for the biperiodization and relaxation for a 2-TL SISL SLAM like HARMONIE.

\section{a. Mathematical description}

In Boyd (2005), the author presents a new approach to create a periodic field from a nonperiodic one. Figure 3 gives a schematic illustration of this procedure in the 1D case. For convenience, we use the same notations as in that paper. Let us consider a nonperiodic function $f$, which is to be periodized on the interval $[-\Theta, \Theta]$. The physical domain is limited to the smaller interval $[-\chi, \chi]$. The intervals $E_{L}=[-\Theta,-\chi]$ and $E_{R}=[\chi, \Theta]$ constitute the extension zone that will be filled such as to render a periodic function.

Let us now define a windowing function $\mathcal{B}$ with the following characteristics: $\mathcal{B}=1$ on the physical domain 
$[-\chi, \chi] ; \mathcal{B}=0$ outside the interval $[\chi-2 \Theta, 2 \Theta-\chi]$; all derivatives of $\mathcal{B}$ are zero at $\pm \chi$ and at $\pm(2 \Theta-\chi)$; and $\sum_{k=-\infty}^{\infty} \mathcal{B}(x+2 k \Theta)=1$. Boyd proposes to use the following function for $\mathcal{B}$ :

$$
\mathcal{B}(x)=\left\{\begin{array}{lll}
0 & \text { for } & |x| \geq 2 \Theta-\chi \\
\frac{1}{2}+\frac{1}{2} \operatorname{erf}\left[\frac{L}{2} \frac{(2 \Theta-\chi-|x|)-(|x|-\chi)}{(2 \Theta-\chi-|x|)(|x|-\chi)}\right] & \text { for } \quad \chi<|x|<2 \Theta-\chi, \\
1 & \text { for } \quad|x| \leq \chi
\end{array}\right.
$$

where $L$ is a tunable parameter and $\operatorname{erf}(x)$ is the error function (Olver et al. 2010). This function is shown in Fig. 3.

The periodization of the function $f$ is then done in two steps. First the function is multiplied with the windowing function $\mathcal{B}$. Next, a summation is taken of shifted products:

$$
\tilde{f}(x)=\sum_{k=-\infty}^{\infty} \mathcal{B}(x+2 k \Theta) f(x+2 k \Theta) .
$$

In practice, only the terms for $k=-1,0,1$ will contribute to $\tilde{f}$ in the periodization interval $[-\Theta, \Theta]$.

This periodization procedure requires information from outside the periodization interval, since the windowing function is nonzero in the intervals $G_{L}=(\chi-$ $2 \Theta,-\Theta]$ and $G_{R}=[\Theta, 2 \Theta-\chi)$ (see Fig. 3). However, as noted by Boyd, this does not pose any problems since the periodization is done on data from the host model.

Another aspect of Boyd's proposal concerns the relaxation procedure. Indeed, Boyd suggests that the same windowing function is used as relaxation function $\alpha$, and that the Davies relaxation zone (referred to before as I zone) can be overlapped with the extension zone. Figure 4 gives a schematic representation of the different parts of the domain in this configuration.

\section{b. Use of the windowing-based method in HARMONIE}

Comparing the domain organization proposed by Boyd (Fig. 4) with the domain organization of HARMONIE (Fig. 2), one comes to the conclusion that they are quite similar. Both approaches provide an extension zone for periodization purposes, and both use Davies relaxation for coupling to the large-scale host model. The HARMONIE system therefore is a suitable candidate to test Boyd's proposal in an NWP context.

However, some aspects deserve closer attention. As outlined in section 2, HARMONIE uses a semiLagrangian approach for the advection terms. This means that time steps are taken that are considerably larger than the CFL limit. Therefore, the claim that the periodization and relaxation do not produce any errors does not hold, since the semi-Lagrangian scheme can propagate errors quickly and deeply into the physical domain. To limit this effect, we will consider in this paper a modified domain organization that still uses the windowing-based periodization procedure, but where the $I$ zone and the $E$ zone do not overlap. Figure 5 gives a schematic representation of this domain organization. In Part II, NWP experiments are carried out with an overlapping $I$ zone and $E$ zone.

As indicated in section $2 \mathrm{a}$, all periodization of the fields in HARMONIE is done during the preparation of the initial and LBC data. Since this is done based on data from a global model, the fact that the windowing-based periodization method requires data from outside the $(C+I+E)$ zone (viz., from the $G_{L}$ and $G_{R}$ zones, see Fig. 5) does not pose any difficulty.

It remains an open question whether the truncation of the SL trajectories at the edge of the $(C+I)$ zone is still necessary when using the windowing-based periodization method. Indeed, this method ensures that the fields are still near physical in the immediate vicinity of this edge, as opposed to the spline-based method, which fills the $E$ zone with completely artificial data. However, when the time step is too large, the SL trajectories will penetrate too far into the $E$ zone, and unphysical information may still enter the $(C+I)$ zone.

Finally, the fact that the windowing-based method ensures infinite-order continuity at the edge of the $E$ zone ensures a fast convergence of the Fourier spectrum. This property is especially interesting for HARMONIE, since all spectral fields are elliptically truncated in spectral

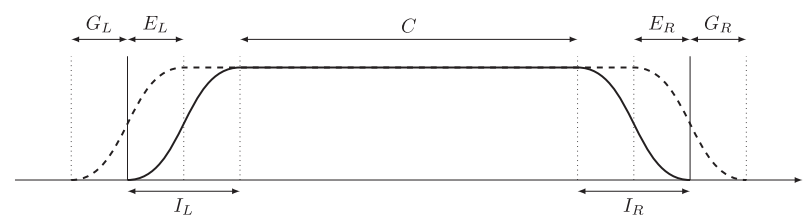

FIG. 4. Organization of the domain in Boyd's proposal: central zone $C$, coupling zone $I=I_{L} \cup I_{R}$, extension zone $E=E_{L} \cup E_{R}$, and additional zone in global model $G=G_{L} \cup G_{R}$. The coupling zone and the extension zone overlap. The solid line represents the relaxation function $\alpha$, the dashed line represents the windowing function $\mathcal{B}$. 


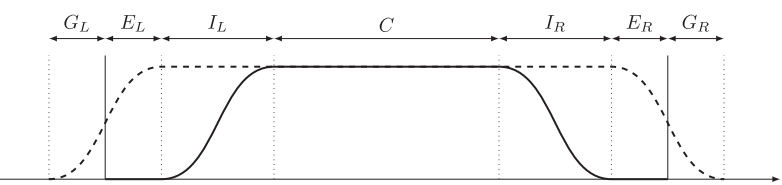

FIG. 5. Organization of the domain when using Boyd's proposal without the overlap: central zone $C$, coupling zone $I=I_{L} \cup I_{R}$, and extension zone $E=E_{L} \cup E_{R}$. The solid line represents the relaxation function $\alpha$, the dashed line represents the windowing function $\mathcal{B}$.

space. In a 2D Fourier spectral space, waves along an oblique direction can have a shorter wavelength than the waves along the axis directions. To obtain an isotropic representation of the fields, those waves are removed. To limit the effect of this spectral truncation, a quickly decaying Fourier spectrum is essential.

\section{c. The different $L B C$ configurations implemented within the HARMONIE system}

Given the conclusions regarding the spectral 2-TL SISL nature of the dynamics with long times steps, as described in the previous subsections it has been decided to implement the following four options in the code:

- the option between using, for the biperiodization, the splines (as described in section 2a and the appendix), denoted by $\mathrm{S}$ or the windowing-based method described in section 3 a denoted by B;

- the option for the Davies relaxation function between the old $\alpha$ function defined in Eq. (4) and denoted by A or using the erf-based $\mathcal{B}$ defined in Eq. (5) denoted by $\mathrm{E}$;

- the option to have the an overlap between the Davies relaxation as proposed by Boyd (2005) (and shown in Fig. 4) denoted by $\mathrm{O}$ or the a disjoint split between the relaxation in the $I$ zone and the windowing in the $E$ zone as shown in Fig. 5 denoted by $S$;

- the option to apply trajectory truncation at the edge of the $(C+I)$ zone denoted by $\mathrm{T}$ or no such trajectory truncation denoted by $\mathrm{N}$.

The HARMONIE system presently can run with any combination of the above four options. The old configuration implemented in the ALADIN model in the past is the SAST configuration. The configuration for the Eulerian scheme in the paper of Boyd (2005) can be described as BEO. From the arguments provided above, the recommended coupling configurations are, a priori, BESN or BASN. Table 1 summarizes these lateral-boundary configurations, considered in the present paper.

\section{Operational reproducibility tests of the windowing-based method}

Here we present the first tests of the implementation of the windowing-based method in the ALARO model, which is part of the physics options within the HARMONIE system. This model is running operationally at the Royal Meteorological Institute of Belgium (RMI). This model is running with the HPE version of the above-mentioned ALADIN dynamical kernel. The primary goal of this section is to examine the operational viability of using Boyd's proposal inside ALARO. We restrict ourselves here to the following question: if we replace our current spline-based method by the windowing-based method, will the result be neutral in terms of scores?

The experiments in this section are therefore designed to mimic the operational forecasts over the domain presented in Fig. 1. It is a regular grid on a Lambert projection, with its center at $\left(50.57^{\circ} \mathrm{N}, 4.55^{\circ} \mathrm{E}\right)$, with 180 physical grid points in the east-west and north-south directions. As shown in Fig. 1, an extra $E$ zone of six points in all directions is added for the biperiodization of the fields. The domain is vertically discretized with 46 hybrid pressure terrain-following levels. The height of the lowest layer center is about $17 \mathrm{~m}$ above the ground. The time step used in these experiments is $300 \mathrm{~s}$. The lateral boundary conditions data are provided by the ALADIN version running at Météo-France. The coupling update frequency is $3 \mathrm{~h}$, which is the same as in the operational setup.

A series of simulations has been performed for two months (January 2010 and July 2010), using the original spline-based periodization procedure denoted as ALRSAST, and using the windowing-based method with and without truncation of the SL trajectories at the boundaries. Here, we only present the configuration with

TABLE 1. Overview of the different lateral-boundary coupling configurations considered in the present study.

\begin{tabular}{llccc}
\hline \hline Configuration & Biperiodicity & Davies relaxation & $\begin{array}{c}\text { Overlap between relaxation } \\
\text { and periodization }\end{array}$ & SL truncation \\
\hline SAST & Splines & $\alpha$ & Split & Truncation \\
BEO & Boyd's periodicity & erf & Overlap & No truncation \\
BASN & Boyd's periodicity & $\alpha$ & Split & Truncation \\
BAST & Boyd's periodicity & $\alpha$ & Split & \\
\hline
\end{tabular}


10m Wind speed BIAS and RMSE (January 2010): UCCLE

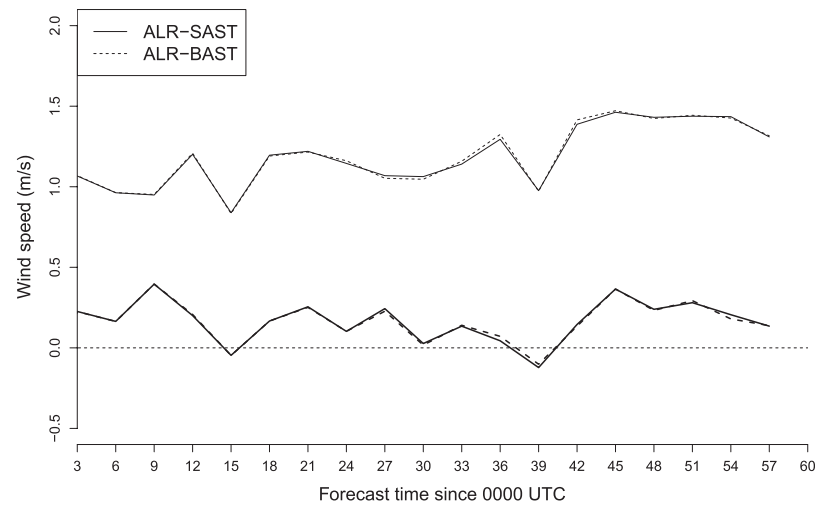

2m Temperature BIAS and RMSE (January 2010): UCCLE

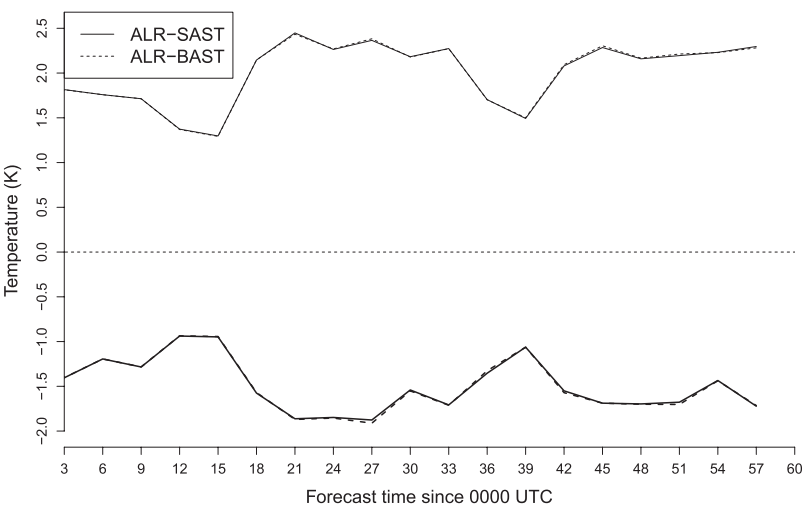

Surface Pressure BIAS and RMSE (January 2010): UCCLE

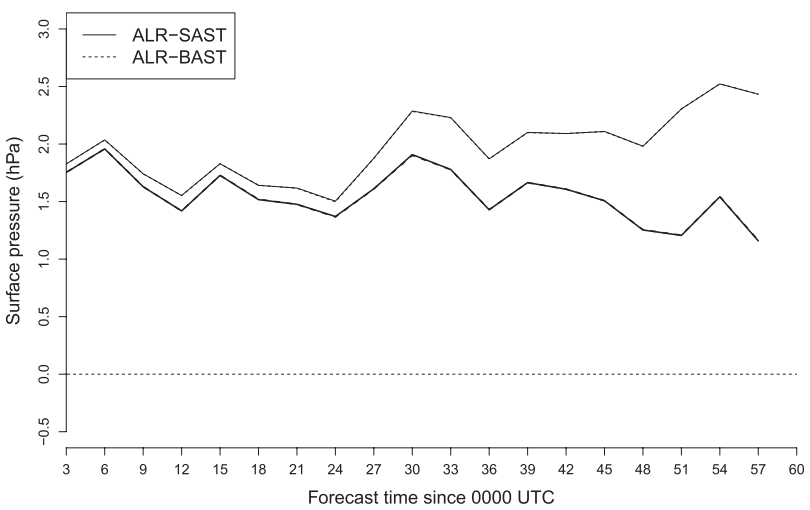

10m Wind speed BIAS and RMSE (July 2010): UCCLE

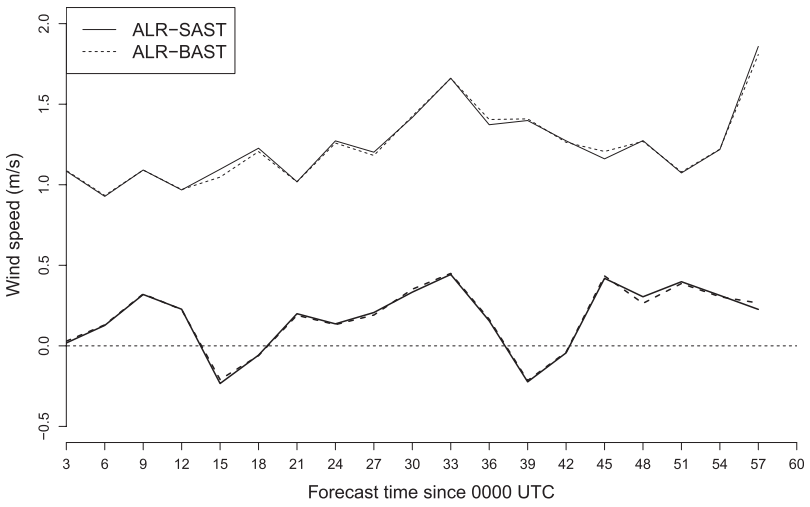

2m Temperature BIAS and RMSE (July 2010): UCCLE

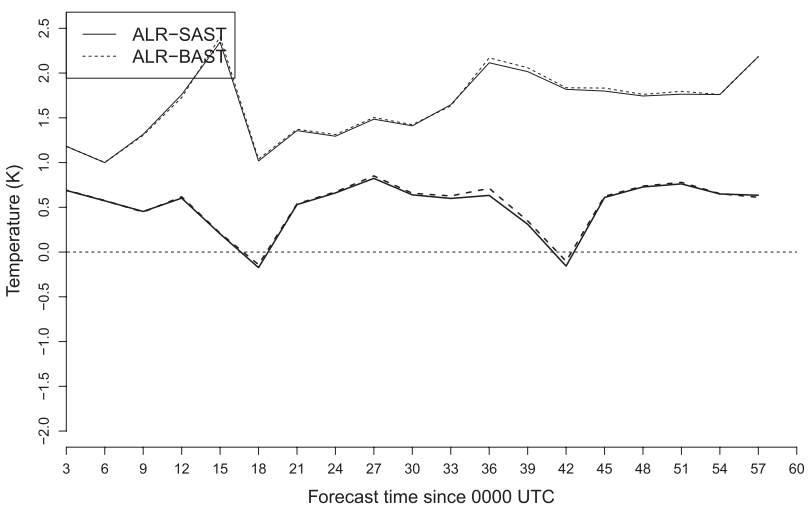

Surface Pressure BIAS and RMSE (July 2010): UCCLE

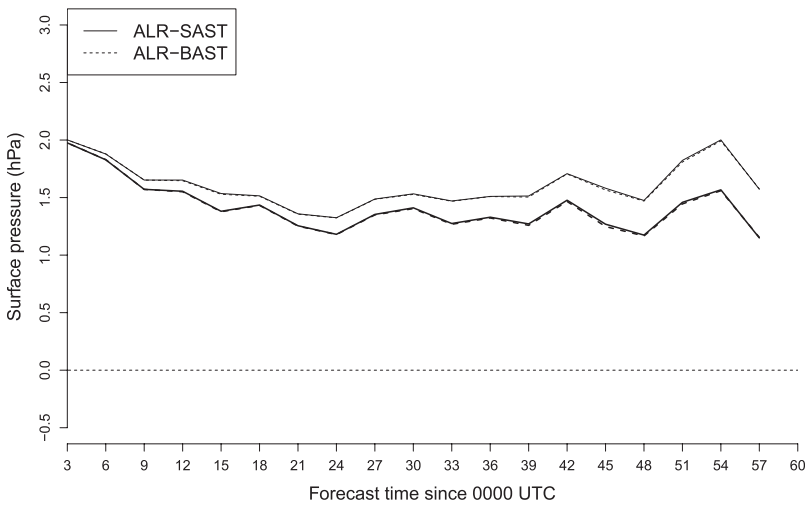

FIG. 6. Results of the reproducibility test with ALARO comparing the windowing-based configuration ALR-BAST to the spline method ALR-SAST. The scores shown are the bias (upper curves) and the rmse (lower curves). Both configurations use the Aladin relaxation function, use separate extension and relaxation zones, and truncate the semi-Lagrangian trajectories.

truncation, denoted as ALR-BAST. The model is run for $60 \mathrm{~h}$ every day of the two months in these three modi. The forecasts are compared to observations every $3 \mathrm{~h}$ at the Uccle observation station, located about $6 \mathrm{~km}$ south of the Brussels city center. The calculated statistical scores are the bias and the root-mean-square error (rmse) between the model output and the observations for all simulations (31 in January and 31 in July).
In Fig. 6 the comparison is made for the 2-m temperature, the $10-\mathrm{m}$ wind speed, and the surface pressure. As can be seen from this figure, the use of ALR-BAST has a neutral impact on the scores (bias and rmse) of these fields. To get a more objective idea of the statistical significance of the differences, confidence intervals are computed with bootstrap techniques (Wilks 1995). These confidence intervals are calculated by resampling 

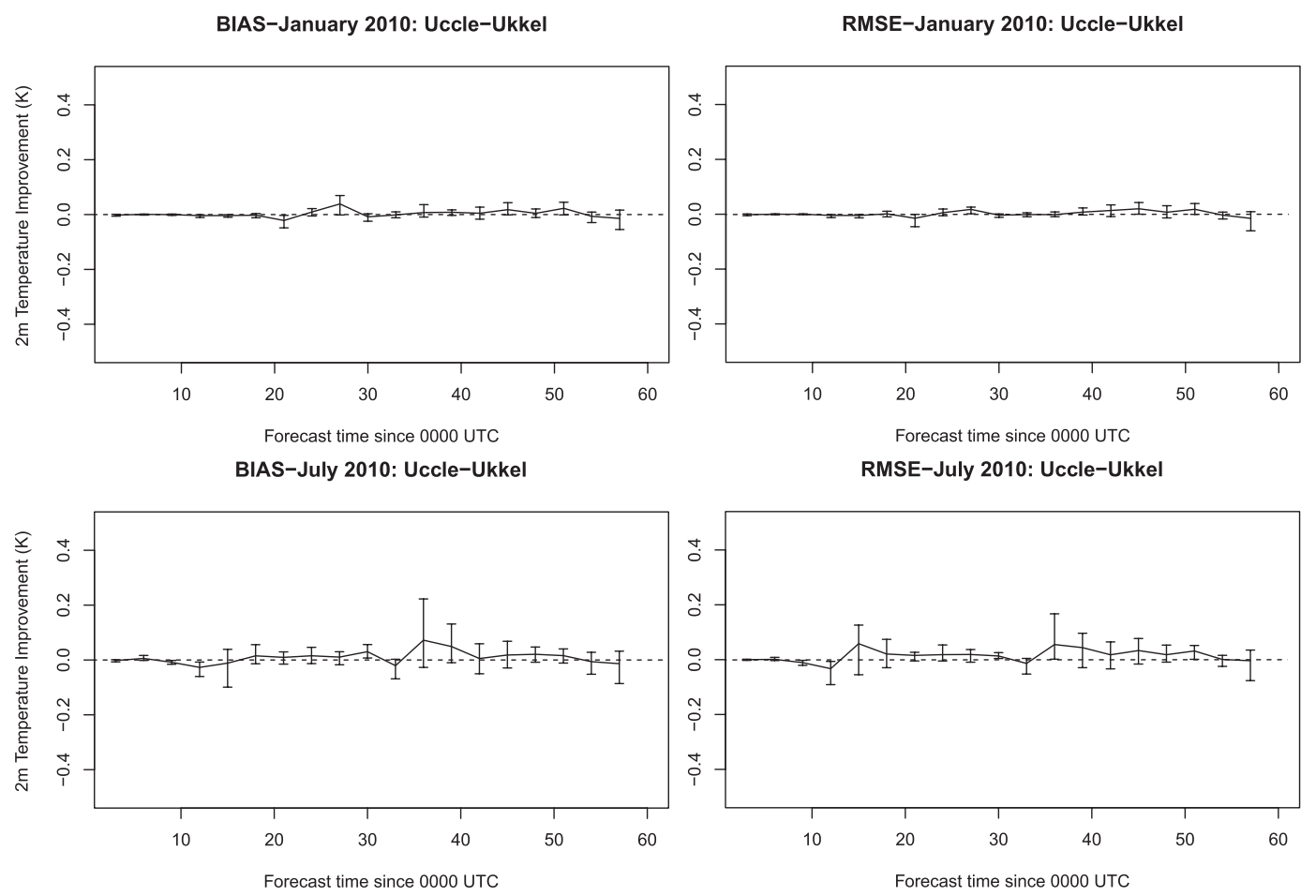

FIG. 7. Statistical significance of the difference between the 2-m temperature bias between the windowing-based configuration ALR-BAST and the spline method ALR-SAST.

the 31-fold samples, for January and July 1000 times and taking the 2.5th and the 97.5th percentiles of $\mid$ bias $_{\text {ALR-BAST }}|-|$ bias $_{\text {ALR-SAST }} \mid$ as lower and upper values to get a $95 \%$ confidence interval for the difference. This means for instance testing the null hypothesis: "the difference of two biases is negative and therefore there is an improvement when using Boyd's proposal."

Figure 7 shows the improvement in the bias of the 2-m temperature obtained when using ALR-BAST instead of ALR-SAST. The difference between the two simulations is therefore not statistically significant. The same result was found for other model fields (not shown here). The forecasts have also been verified with other observation stations across Belgium, yielding the same conclusions.

\section{Discussion and conclusions}

In this paper we present the implementation of the periodization and relaxation procedure proposed by Boyd (2005), specifically for the use in the three-dimensional spectral LAM models of the HARMONIE system. The main advantages of this windowing-based method are the use of physical data for the periodic extension and the excellent spectral convergence. However, the idea put forth in Boyd (2005) of having a spatial overlap between the Davies relaxation and the biperiodization is not recommended in a semi-Lagrangian model when the time step is a few times larger than the Courant number.

An operational-like experiment was performed to test this alternative periodization method, but with a distinct split between Davies relaxation entirely in the so-called $I$ zone and the windowing periodicity in the $E$ zone. Compared to the traditional spline-based approach that existed before in the code of the HARMONIE system, the observational scores show no significant differences. This can be regarded as a reproducibility test indicating that it is safe to replace the old coupling configuration by the windowing-based one in an operational context.

The real improvements are expected to manifest themselves in specific cases of strong dynamical forcing at the lateral boundaries (e.g., for incoming storms). Since such cases are relatively rare events in operational forecast of LAMs, one cannot expect to detect such improvements in averaged forecast scores. In fact, one should rely on so-called perfect-model experiments (de Elía et al. 2002), to avoid drawing erroneous conclusions due to the fact that the lateral-boundary-condition errors could be compensated by model errors other than the ones originating from the lateral-boundary conditions. Also, any improvements due to more accurate boundary conditions have to be compared to the errors originating from the temporal resolution problem (Termonia et al. 2009). So some tests should be 
preferably carried out with coupling updates from the host model at each time step. Such investigations lie outside the scope of the present paper. The accompanying paper (Part II) describes a clean experimental setup addressing these issues and demonstrates the superiority of the windowing-based method in such rare but meteorologically highly relevant cases.

Acknowledgments. We thank Filip Váña for useful discussions and for providing stimulating and pleasant working conditions in the Czech Meteological Institute (CHMI) in Prague where a major part of this work was carried out. We also thank the two anonymous reviewers for their valuable comments.

\section{APPENDIX}

\section{Details of the Periodization by Splines in the HARMONIE Code}

Let us denote the discretized field as $F_{I}$ with $I=1, \ldots$, $N$ where $N$ indicates the last point of the whole $(C+I+$ $E$ ) zone and call $M$ the location of the last points of the $(C+I)$ zone. Given values of $F_{1}, F_{2}, \ldots, F_{M}$ in the $(C+$ I) zone, the aim is to fill the grid points $M+1, M+2, \ldots$, $N$ with a continuous and periodic function. This is done by the following spline function:

$$
F(M+z)=A_{0}+A_{1} z+A_{2} z^{2}+A_{3} z^{3},
$$

where

$$
\begin{aligned}
& A_{0}=F_{M}, \\
& A_{1}=\frac{F_{1}-F_{M}}{K}-\frac{K}{6}\left(2 D_{M}+D_{1}\right), \\
& A_{2}=\frac{1}{2} D_{M}, \\
& A_{3}=\frac{D_{1}-D_{M}}{6 K},
\end{aligned}
$$

where $K=N-M+1$ is the width of the $E$ zone expressed in gridpoint distances and

$$
\begin{aligned}
D_{1} & =\frac{3}{2+\lambda} \frac{2 d_{1}-\lambda d_{M}}{2-\lambda}, \\
D_{M} & =\frac{3}{2+\lambda} \frac{2 d_{M}-\lambda d_{1}}{2-\lambda},
\end{aligned}
$$

with $\lambda=K /(K+1)$. These are in fact smoothed versions of the following estimates of the second-order derivative in the points 1 and $M$ :

$$
\begin{aligned}
d_{M} & =\frac{2}{K+1}\left(F_{M-1}-F_{M}+\frac{F_{1}-F_{M}}{K}\right), \\
d_{1} & =\frac{2}{K+1}\left(F_{2}-F_{1}+\frac{F_{M}-F_{1}}{K}\right) .
\end{aligned}
$$

It can be easily seen that this spline satisfies the condition of continuity in the points 1 and $M$ and the following conditions on the second-order derivatives in point 1 and $M: F^{\prime \prime}(M)=D_{M}$ and $F^{\prime \prime}(N+1)=D_{1}$.

In the HARMONIE code, these splines are applied in both the $x$ direction and the $y$ direction of the domain. The resulting two-dimensional fields are then additionally smoothed in the $E$ zone by the following filter:

$$
\begin{aligned}
F_{I, J}^{\text {smooth }}= & \frac{1}{4} F_{I, J}+\frac{1}{8}\left(F_{I+1, J}+F_{I-1, J}+F_{I, J+1}+F_{I, J-1}\right) \\
& +\frac{1}{16}\left(F_{i+1, j+1}+F_{i+1, j-1}+F_{i-1, j+1}+F_{i-1, j-1}\right) .
\end{aligned}
$$

\section{REFERENCES}

ALADIN International Team, 1997: The ALADIN project: Mesoscale modelling seen as a basic tool for weather forecasting and atmospheric research. WMO Bull., 46, 317-324.

Bénard, P., J. Vivoda, J. Másek, P. Smolíková, K. Yessad, C. Smith, R. Brožková, and J.-F. Geleyn, 2010: Dynamical kernel of the Aladin-NH spectral limited-area model: Revised formulation and sensitivity experiments. Quart. J. Roy. Meteor. Soc., 136, $155-169$.

Boyd, J. P., 2002: A comparison of numerical algorithms for Fourier extension of the First, Second and Third kinds. J. Comput. Phys., 178, 118-160.

_ 2005: Limited-area Fourier spectral models and data analysis schemes: Windows, Fourier extension, Davies relaxation, and all that. Mon. Wea. Rev., 133, 2030-2042.

Bubnová, R., G. Hello, P. Bénard, and J.-F. Geleyn, 1995: Integration of the fully elastic equations cast in the hydrostatic pressure terrain-following coordinate in the framework of the ARPEGE/Aladin NWP system. Mon. Wea. Rev., 123, 515535.

Catry, B., J.-F. Geleyn, M. Tudor, P. Bénard, and A. Trojáková, 2007: Flux-conservative thermodynamic equations in a massweighted framework. Tellus, 59A, 71-79.

Davies, H. C., 1976: A lateral boundary formulation for multilevel prediction models. Quart. J. Roy. Meteor. Soc., 102, 405-418.

_ 1983: Limitations of some common lateral boundary schemes used in regional NWP models. Mon. Wea. Rev., 111, 10021012

de Elía, R., R. Laprise, and B. Denis, 2002: Forecasting skill limits of nested, limited-area models: A perfect-model approach. Mon. Wea. Rev., 130, 2006-2023.

Degrauwe, D., S. Caluwaerts, F. Voitus, R. Hamdi, and P. Termonia, 2012: Application of Boyd's periodization and relaxation method in a spectral atmospheric limited-area model. Part II: Accuracy analysis and detailed study of the operational impact. Mon. Wea. Rev., 140, 3149-3162. 
Geleyn, J.-F., B. Catry, Y. Bouteloup, and R. Brožková, 2008: A statistical approach for sedimentation inside a micro-physical precipitation scheme. Tellus, 60A, 649-662.

Gerard, L., 2007: An integrated package for subgrid convection, clouds and precipitation compatible with the meso-gamma scales. Quart. J. Roy. Meteor. Soc., 133, 711-730.

— tion parameterization in a limited area model with increasing resolution. Quart. J. Roy. Meteor. Soc., 131, 2293-2312.

— J.- J.M. Piriou, R. Brožková, J.-F. Geleyn, and D. Banciu, 2009: Cloud and precipitation parameterization in a meso-gammascale operational weather prediction model. Mon. Wea. Rev., 137, 3960-3977.

Gustafsson, N., L. Berre, S. Hörnquist, X.-Y. Huang, M. Lindskog, B. NavascuÉs, S. Mogensen, and S. Thorsteinsson, 2001: Three-dimensonal variational data assimilation for a limited area model. Part I: General formulation and the background error constraint. Tellus, 53A, 425-446.

Hamdi, R., H. V. de Vyver, and P. Termonia, 2012: New cloud and microphysics parameterization for use in high-resolution dynamical downscaling: Application for summer extreme temperature over Belgium. Int. J. Climatol., in press.

Haugen, J. E., and B. Machenhauer, 1993: A spectral limited-area model formulation with time-dependent boundary conditions applied to the shallow-water equations. Mon. Wea. Rev., 121, 2618-2630.

Hortal, M., 2002: The development and testing of a new two-timelevel semi-Lagrangian scheme (SETTLS) in the ECMWF forecast model. Quart. J. Roy. Meteor. Soc., 128, 1671-1687.

Hoyer, J. M., 1987: The ECMWF spectral limited area model. Proc. ECMWF Workshop on Techniques for Horizontal Discretization in Numerical Weather Prediction Models, Reading, United Kingdom, ECMWF, 343-359.

Juang, H., 1992: A spectral fully compressible nonhydrostatic mesoscale model in hydrostatic sigma coordinates: Formulation and preliminary results. Meteor. Atmos. Phys., 50, 75-88.

Kuo, H.-C., and R. T. Williams, 1992: Boundary effects in regional spectral models. Mon. Wea. Rev., 120, 2986-2992.

Laprise, R., 1992: The Euler equations of motion with hydrostatic pressure as an independent variable. Mon. Wea. Rev., 120, 197-207.

- 2003: Resolved scales and nonlinear interactions in limitedarea models. J. Atmos. Sci., 60, 768-779.

Olver, F., D. Lozier, R. Boisvert, and C. Clark, Eds., 2010: NIST Handbook of Mathematical Functions. Cambridge University Press, 968 pp.

Radnóti, G., 1995: Comments on "A spectral limited-area formulation with time-dependent boundary conditions applied to the shallow-water equations." Mon. Wea. Rev., 123, 3122-3123.
Radu, R., M. Déqué, and S. Somot, 2008: Spectral nudging in a spectral regional climate model. Tellus, 60A, 898-910.

Ritter, B., and J.-F. Geleyn, 1992: A comprehensive radiation scheme for numerical weather prediction models with potential applications in climate simulations. Mon. Wea. Rev., 120, 303-325.

Seity, Y., P. Brousseau, S. Malardel, G. Hello, P. Bénard, F. Bouttier, C. Lac, and V. Masson, 2011: The AROMEFrance convective-scale operational model. Mon. Wea. Rev., 139, 976-991.

Simmons, A. J., and D. M. Burridge, 1981: An energy and angularmomentum conserving vertical finite-difference scheme and hybrid vertical coordinates. Mon. Wea. Rev., 109, 758-766.

Tatsumi, Y., 1986: A spectral limited-area model with timedependent lateral boundary conditions and its application to a multi-level primitive equation model. J. Meteor. Soc. Japan, 64, 637-663.

Temperton, C., M. Hortal, and A. Simmons, 2001: A two-timelevel semi-Lagrangian global spectral model. Quart. J. Roy. Meteor. Soc., 127, 111-126.

Termonia, P., 2003: Monitoring and improving the temporal interpolation of lateral-boundary coupling data for limited-area models. Mon. Wea. Rev., 131, 2450-2463.

_ 2004: Monitoring the coupling-update frequency of a limitedarea model by means of a recursive digital filter. Mon. Wea. Rev., 132, 2130-2141.

— conditions from the dynamic core in semi-implicit semiLagrangian models. Tellus, 60A, 632-648.

—, A. Deckmyn, and R. Hamdi, 2009: Study of the lateral boundary condition temporal resolution problem and a proposed solution by means of boundary error restarts. Mon. Wea. Rev., 137, 3551-3566.

_ D. Degrauwe, and R. Hamdi, 2011: Improving the temporal resolution problem by localized gridpoint nudging in regional weather and climate models. Mon. Wea. Rev., 139,1292-1304.

Tudor, M., and P. Termonia, 2010: Alternative formulations for incorporating lateral boundary data into limited-area models. Mon. Wea. Rev., 138, 2867-2882.

Váña, F., P. Bénard, J.-F. Geleyn, A. Simon, and Y. Seity, 2008: Semi-Lagrangian advection scheme with controlled damping: An alternative to nonlinear horizontal diffusion in a numerical weather prediction model. Quart. J. Roy. Meteor. Soc., 134, 523-537, doi:10.1002/qj.220.

Voitus, F., P. Termonia, and P. Bénard, 2009: Well-posed lateral boundary conditions for spectral semi-implicit semi-Lagrangian schemes: Tests in a one-dimensional model. Mon. Wea. Rev., 137, 315-330.

Wilks, D. S., 1995: Statistical Methods in the Atmospheric Sciences: An Introduction. Academic Press, 467 pp. 